\title{
Quantifying Fusion Born Ion Populations in Magnetically Confined Plasmas using Ion Cyclotron Emission
}

\author{
L. Carbajal, ${ }^{1,2, *}$ R. O. Dendy, ${ }^{2,3}$ S. C. Chapman, ${ }^{2}$ and J. W. S. Cook ${ }^{2,4}$ \\ ${ }^{1}$ Oak Ridge National Laboratory, PO Box 2008, Oak Ridge, Tennessee 37831-6169, USA \\ ${ }^{2}$ Centre for Fusion, Space and Astrophysics, Department of Physics, Warwick University, Coventry CV4 7AL, United Kingdom \\ ${ }^{3}$ CCFE, Culham Science Centre Abingdon, Oxfordshire OX14 3DB, United Kingdom \\ ${ }^{4}$ First Light Fusion Ltd., Oxford Industrial Park, Yarnton, Oxfordshire OX5 1QU, United Kingdom
}

(Received 8 June 2016; revised manuscript received 27 January 2017; published 7 March 2017)

\begin{abstract}
Ion cyclotron emission (ICE) offers a unique promise as a diagnostic of the fusion born alpha-particle population in magnetically confined plasmas. Pioneering observations from JET and TFTR found that ICE intensity $P_{\mathrm{ICE}}$ scales approximately linearly with the measured neutron flux from fusion reactions, and with the inferred concentration, $n_{\alpha} / n_{i}$, of fusion born alpha particles confined within the plasma. We present fully nonlinear self-consistent kinetic simulations that reproduce this scaling for the first time. This resolves a long-standing question in the physics of fusion alpha-particle confinement and stability in magnetic confinement fusion plasmas. It confirms the magnetoacoustic cyclotron instability as the likely emission mechanism and greatly strengthens the basis for diagnostic exploitation of ICE in future burning plasmas.
\end{abstract}

DOI: 10.1103/PhysRevLett.118.105001

The containment of populations of alpha particles, born in nuclear fusion reactions between thermal ions, is central to the exploitation of magnetically confined plasmas for energy generation. In contrast to the thermal (10 to $20 \mathrm{keV})$ deuterium and tritium ions that form the bulk of a burning plasma, alpha particles are born at $3.5 \mathrm{MeV}$ and in consequence are only marginally confined even in the largest contemporary tokamak experiment, JET. These alpha particles must remain confined while they transfer energy, via collisions with electrons, to the bulk plasma on a slowing-down time scale $\sim 0.5 \mathrm{~s}$ which is of order $10^{7}$ gyroperiods. The alpha particles also couple noncollisionally to the thermal plasma and magnetic field, for example, by fast time scale collective resonant interaction with magnetohydrodynamics (MHD) modes. A key objective of ITER is to explore the confinement physics of fusion born alpha particles under conditions where, unlike at present, they are the primary heating source for the plasma. Direct observation of the spatial distribution and collective dynamics of this alpha-particle population is challenging: they are only $\sim 0.1 \%$ of the plasma particles, their singleparticle radiation is in the background, and in situ probe measurements are impossible.

Ion cyclotron emission (ICE) offers unique promise as a diagnostic of the alpha particles, and has been proposed for this role in future deuterium-tritium plasmas in JET and ITER [1-3]. Pioneering observations from JET and TFTR [4-6] found that ICE intensity $P_{\text {ICE }}$ scales approximately linearly with the measured neutron flux from fusion reactions, and with the inferred concentration, $n_{\alpha} / n_{i}$, of fusion born alpha particles confined within the plasma. Understanding the physical basis for this scaling is important because, in future experiments with burning plasma, measurements of the observed ICE intensity could then directly diagnose the number density, orbital characteristics, and temporal evolution of the confined alpha-particle population. From its suprathermal nature, it is evident that ICE arises from a collective radiative instability, and the leading candidate is the magnetoacoustic cyclotron instability (MCI) [7-13]. The MCI is driven by the free energy of the alpha particles, whose distribution in velocity space can exhibit a natural population inversion, localized in space at the outer midplane edge of the plasma. The way in which MCI saturation physics leads to the observed scaling of $P_{\text {ICE }}$ with $n_{\alpha} / n_{i}$ has not hitherto been determined. In particular, the lower cyclotron harmonics $(1 \leq l \leq 6)$ are typically linearly stable against the MCI; hence, their existence and magnitude are solely determined by nonlinear physics $[14,15]$. We have therefore performed kinetic numerical simulations of the MCI that are fully nonlinear and incorporate self-consistent particle dynamics and field evolution. Identifying the saturated intensity of the simulated MCI-excited fields with $P_{\mathrm{ICE}}$, we are able to reproduce the observed approximately linear scaling of $P_{\mathrm{ICE}}$ with $n_{\alpha} / n_{i}$. These first-principles simulations also enable us to directly identify the underlying physics of the observed scaling.

In the early JET $D$ - $T$ experiments [5,6,16,17], high power neutral beam injection (NBI) was used to heat the plasma. By comparing plasmas where only deuterium was used as the NBI source to those where a mixture of deuterium and tritium was used, a linear correlation was identified between the measured ICE power at the second cyclotron harmonic $\omega=2 \Omega_{\alpha}$ and the measured fusion reactivity, for experiments spanning a factor of a million in the neutron source rate (cf., Fig. 3 of Ref. [6]). In 
addition, time resolved measurements during specific JET $D-T$ plasmas show linear scaling of ICE intensity at $\omega=$ $2 \Omega_{\alpha}$ with local edge alpha-particle concentration as time evolved (cf., Fig. 4 of Ref. [6]). These results strongly suggest that the source of ICE in those plasmas was the fusion born alpha particles resulting from $D-T$ fusion reactions. Furthermore, in all these large tokamak plasmas, the ICE power spectrum displayed peaks at sequential cyclotron harmonics of alpha particles at the outer midplane edge. These characteristics are largely replicated by recent second generation ICE measurements, to which the work reported here is also relevant. ICE is used as a diagnostic for energetic ions lost from $D I I I-D$ tokamak plasmas due to MHD activity [18]. ICE is observed from ASDEX-Upgrade [19,20] and JT-60U [21,22] tokamak plasmas, with spectral peaks at edge cyclotron harmonics of ion species that include the energetic products of fusion reactions in pure deuterium, namely, protons, tritons, and helium-3 ions. ICE from sub-Alfvénic beam ions, injected at tens of $\mathrm{keV}$ to heat the plasma, was observed from TFTR tokamak plasmas, and is also interpreted in terms of the MCI [23]. ICE that is probably of this kind is also used as a diagnostic of lost fast ions in large stellarator-heliotron plasmas in large helical device (LHD) [24]. Very recently, ICE with spectral peak separation equal to the edge proton cyclotron frequency has been reported from deuterium plasmas in the KSTAR tokamak [25].

Radiation similar to ICE is also observed in space plasmas, driven by energetic ions with drifting ringlike velocity distributions, and has also been interpreted in terms of the MCI [26,27]. Its nonlinear development can be resolved in fully kinetic simulations, for example, in reforming perpendicular shocks in space plasmas that show clear spectral peaks at consecutive ion cyclotron harmonics, suggesting the occurrence of ICE in the region behind the shock front [28]. Fully self-consistent kinetic simulations of the MCI may thus be relevant to ICE occurring in space as well as fusion plasmas [14,15]. ICE phenomenology could also be exploited for "alpha channeling" in magnetic confinement fusion (MCF) plasmas [29].

In this Letter we simulate the MCI in the hybrid approximation in which the ion dynamics (both energetic alpha particles and majority thermal ions) follow the full Lorentz force, and the electrons are represented as a fluid. Our simulations self-consistently evolve the low-frequency Maxwell-Lorentz system for all three vector components of the particle velocities and electric and magnetic fields, in one spatial dimension. We study the MCI for concentrations of alpha particles relative to thermal ions that range between $n_{\alpha} / n_{i}=10^{-4}$ and $10^{-3}$. The lower value is the smallest that is realizable, using reasonable computational resources, while carrying the MCI into its nonlinear regime, which starts at $15 \tau_{\alpha}$ and extends up to $30 \tau_{\alpha}$ for this alpha-particle concentration. At higher concentrations, the nonlinear MCI takes over at earlier times. Here $\tau_{\alpha}$ is the alpha-particle gyroperiod. Previous studies [5,6], together with our calculations of alpha-particle orbits in JET-like axisymmetric magnetic fields, show that the transit time within the outer midplane plasma of alpha particles driving ICE can be as long as $60 \tau_{\alpha}$, giving enough time for the nonlinear MCI to be fully developed.

We demonstrate that the physical basis for the observed approximately linear scaling of $P_{\mathrm{ICE}}$ with $n_{\alpha} / n_{i}$ is as follows. The most strongly linearly unstable cyclotron harmonic modes $\omega=l \Omega_{\alpha}(8 \leq l \leq 10)$ grow rapidly, initially at their linear growth rates, which scale as $\sqrt{n}_{\alpha}$. This growth is so rapid that its linear phase terminates after only a few gyroperiods. Beating between these linearly excited modes drives the growth of the lower harmonic $(1 \leq l \leq 6)$ linearly stable modes. Our fully nonlinear simulations show that these energy flows are such that, at saturation, all mode intensities scale approximately with the concentration of alpha particles.

We use the PROMETHEUS++ code [30], which implements the hybrid approximation $[31,32]$ as applied to the MCI in Ref. [15]. In this approximation, the plasma is quasineutral in this low-frequency (Darwin) limit, so that the total number density is $n=n_{e}=\sum Z_{j} n_{j}$, where $n_{e}$ is the electron number density, and $Z_{j}$ and $n_{j}$ are the atomic number and the number density of the species $j$. In all our simulations, the direction of variation is along the $x$ axis, perpendicular to the background magnetic field, $\boldsymbol{B}_{0}=B_{0} z$.

The plasma parameters are similar to those of the $D-T$ plasmas of JET at the outer midplane edge [5], with background magnetic field $B_{0}=2.1 \mathrm{~T}$, total number density $n=1 \times 10^{19} \mathrm{~m}^{-3}$, and electron temperature $T_{e}=1 \mathrm{keV}$. The background population of deuterons is in thermal equilibrium with the fluid electrons, and the local minority alpha-particle population $n_{\alpha}$ is modeled as a ringlike distribution in velocity space $f_{\alpha}\left(v_{\|}, v_{\perp}\right)=$ $\delta\left(v_{\|}\right) \delta\left(v_{\perp}-u_{\perp}\right)$. Here $u_{\perp}$ is the magnitude of the initial perpendicular velocity of the alpha particles, corresponding to the energy of $3.5 \mathrm{MeV}$ at which alpha particles are born in $D-T$ fusion reactions. This experimentally motivated distribution function corresponds to a simplified version of the one used in the analytical theory of the MCI in Ref. [9], and it is observed to capture, in the simplest way, the excess perpendicular energy of more general distribution functions driving the MCI.

The number density is initially constant across the simulation domain, subject to noise fluctuations. As a check against sensitivity to simulation noise, we repeated all simulations using both a uniform random number generator and a "quiet start" [33]. The latter method reduces the small fluctuations in the initial number density $n$, which can provide an extra source of free energy. The periodic simulation domain consists of 8192 grid cells, each of length equal to $r_{D} / \sqrt{2}$, where $r_{D}=v_{T_{D}} / \Omega_{D}$ is the deuteron Larmor radius, $v_{T_{D}}^{2}=k_{B} T_{D} / m_{D}$ is the deuteron thermal velocity, and $\Omega_{D}$ is the deuteron cyclotron 
frequency. In this way, we resolve in detail the gyromotion of both ion species; alpha-particle dynamics are particularly highly resolved, given that $r_{\alpha} / r_{D} \approx 41$. The simulations use 2000 macroparticles per grid cell to represent each ion population.

To compare our hybrid simulations with measured ICE amplitude it is essential to access the fully nonlinear regime. These hybrid simulations of the MCI then recover the observed scaling properties of ICE intensity $P_{\mathrm{ICE}}$ with respect to the concentration of energetic ions $\xi_{\alpha}=n_{\alpha} / n_{i}$, observed in JET, giving further support to the MCI as the mechanism underlying ICE. We perform hybrid simulations of the MCI with twenty different values of $\xi_{\alpha}$, ranging from $\xi_{\alpha}=10^{-4}$ to $\xi_{\alpha}=10^{-3}$. The first result drawn from these simulations is the excitation of perpendicular propagating fast Alfvén waves at consecutive ion cyclotron harmonics of alpha particles, for all the values of $\xi_{\alpha}$. Power spectra of the normalized parallel perturbed magnetic field energy $\delta B_{\|}^{2} / B_{0}^{2}$ for $\xi_{\alpha}=10^{-4}, 10^{-3.5}$, and $10^{-3}$, respectively, are shown in Fig. 1. Noise is reduced in these simulations using a quiet start [33], and the spectral peaks at the cyclotron harmonics emerge well above the noise level. The values chosen for $\xi_{\alpha}$ in Fig. 1 are logarithmically equally spaced. The corresponding spectral peak power for the nonlinearly unstable modes $1 \leq l \leq 6$ can also be seen to increase logarithmically - the relative heights of these peaks are linearly spaced on this log-linear plot. These MCI simulations thus suggest linear scaling of $P_{\mathrm{ICE}}$ with $\xi_{\alpha}$, consistent with experiment.

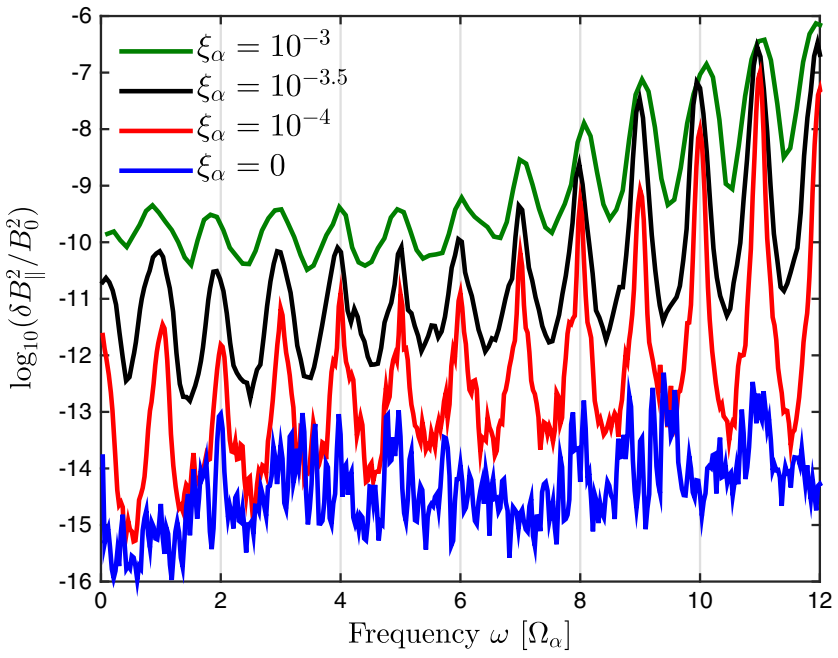

FIG. 1. Log-linear plot of the power spectral density of the normalized perturbed magnetic field $\delta B_{\|} / B_{0}$ in the fully nonlinear regime of the simulations at increasing concentrations $\xi_{\alpha}=$ $n_{\alpha} / n_{i}$ of energetic particles (red, black, and green solid lines). The noise level in the simulations is indicated by the bottom trace (blue solid line), which is a simulation for background plasma only, $\xi_{\alpha}=0$. The values $\xi_{\alpha}$ for the three simulations that include energetic particles are a half decade apart, and the corresponding heights of the peaks in $\log _{10}\left(\delta B_{\|}^{2} / B_{0}^{2}\right)$ can be seen to increase approximately linearly between these traces for harmonics $\leq 6$.
We now examine this in more detail. In Fig. 2 we plot vs $\xi_{\alpha}$ the perturbed normalized magnetic field energy in the fully nonlinear regime of the simulations $\delta B_{\|}^{2} / B_{0}^{2}$ at the peak of each cyclotron harmonic, divided by concentration of energetic ions $\xi_{\alpha}$. On these plots, a straight horizontal trend implies a linear relationship $\delta B_{\|}^{2} / B_{0}^{2} \sim \xi_{\alpha}$. The linearly stable modes $\omega=\Omega_{\alpha}$ to $6 \Omega_{\alpha}$ and the marginally unstable mode $\omega=7 \Omega_{\alpha}$ are plotted in the upper panel (a). The black filled circles in this panel show the measured ICE power $P_{\mathrm{ICE}}$ at $\omega=2 \Omega_{\alpha}$ inferred from Fig. 4 of Ref. [6], the error bars of $P_{\mathrm{ICE}}$ correspond to the uncertainty of $\pm 6 \mathrm{~dB}$ in the detected ICE power reported in Ref. [5]. We can see that there is good correspondence between the observations and the nonlinear peak power at $\omega=\Omega_{\alpha}$ to $6 \Omega_{\alpha}$. The marginally linearly unstable mode at $l=7$ is plotted in both panels (a) and (b) where the ordinates are on different scales. It marks the transition between the linearly stable modes $\omega=\Omega_{\alpha}$ to $6 \Omega_{\alpha}$ and the linearly unstable modes $\omega=7 \Omega_{\alpha}$ to $12 \Omega_{\alpha}$. These linearly unstable modes exhibit approximately linear scaling of intensity with $\xi_{\alpha}$ but with significant scatter. In Fig. 3 we examine the behavior earlier

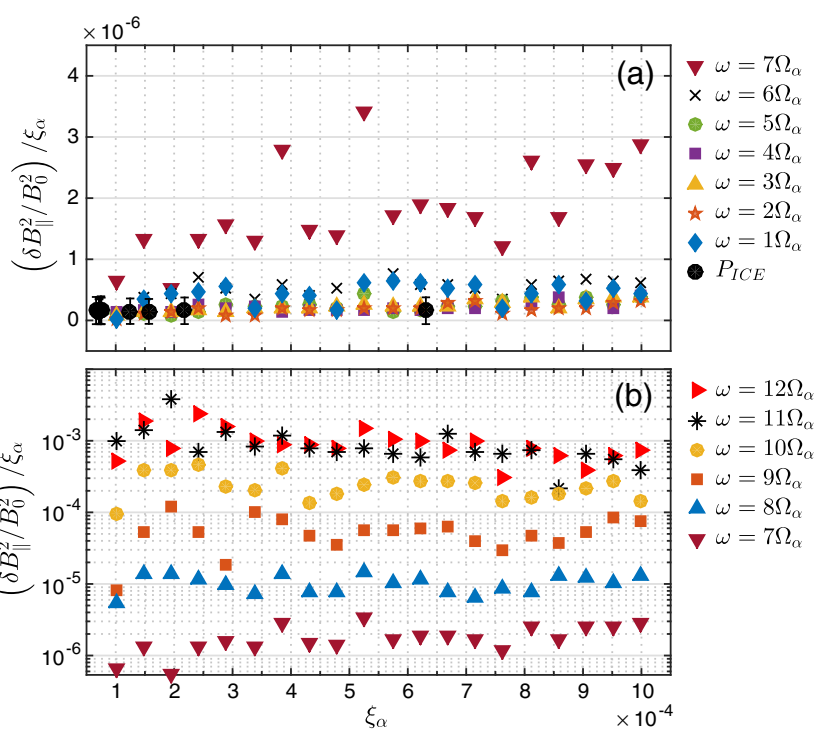

FIG. 2. The perturbed normalized magnetic field energy in the fully nonlinear regime of the simulations $\delta B_{\|}^{2} / B_{0}^{2}$ at the peak of each cyclotron harmonic, divided by concentration of energetic ions $\xi_{\alpha}$, is plotted vs $\xi_{\alpha}$. The linearly stable modes $\omega=\Omega_{\alpha}$ to $6 \Omega_{\alpha}$ and the marginally unstable mode $\omega=7 \Omega_{\alpha}$ are plotted in the upper panel (a). The black filled circles in this panel show the measured ICE power $P_{\text {ICE }}$ at $\omega=2 \Omega_{\alpha}$ inferred from Fig. 4 of Ref. [6]; the error bars of $P_{\text {ICE }}$ correspond to the uncertainty of $\pm 6 \mathrm{~dB}$ in the detected ICE power reported in Ref. [5]. Here, $P_{\text {ICE }}$ has been rescaled to the value of $B_{\|}^{2} / B_{0}^{2}$ at the second harmonic spectral peak for $\xi_{\alpha} \approx 6 \times 10^{-4}$, which is the maximum value of $\xi_{\alpha}$ for which $P_{\text {ICE }}$ is reported in Ref. [5]. The lower panel (b) plots, in the same format, the linearly unstable modes $\omega=7 \Omega_{\alpha}-12 \Omega_{\alpha}$. Note the different scale of the ordinate in the two panels. On these compensated plots, a straight horizontal trend implies a relationship $\delta B_{\|}^{2} / B_{0}^{2} \sim \xi_{\alpha}$. 


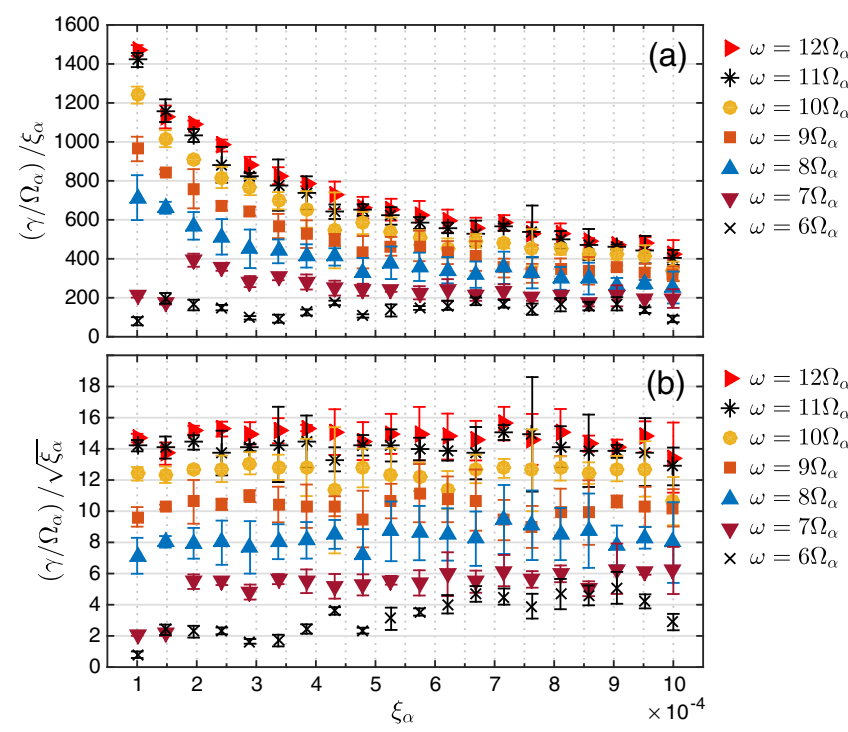

FIG. 3. The growth rate for the MCI obtained at early times in the simulation, $\gamma$, normalized to $\Omega_{\alpha}$ and divided by concentration of energetic ions $\xi_{\alpha}$ [upper panel (a)] and divided by $\sqrt{\xi_{\alpha}}$ [lower panel (b)], are plotted vs $\xi_{\alpha}$ for linearly stable mode $\omega=6 \Omega_{\alpha}$ and linearly unstable modes $\omega=7$ to $12 \Omega_{\alpha}$. On these compensated plots, a straight horizontal trend implies a relationship $\gamma / \Omega_{\alpha} \sim \xi_{\alpha}$ [upper panel (a)] and $\gamma / \Omega_{\alpha} \sim \sqrt{\xi_{\alpha}}$ [lower panel (b)].

in the simulations, during the linear growth phase of the MCI of modes $\omega=7 \Omega_{\alpha}$ to $12 \Omega_{\alpha}$. In this figure, the growth rate for the MCI obtained at early times in the simulation, $\gamma$ (normalized to $\Omega_{\alpha}$ ), is plotted in the upper panel (a) divided by concentration of energetic ions $\xi_{\alpha}$, and in the lower panel (b) divided by $\sqrt{\xi_{\alpha}}$. Figure 3 then tests whether a relationship $\gamma / \Omega_{\alpha} \sim \xi_{\alpha}$ [upper panel (a)] or $\gamma / \Omega_{\alpha} \sim \sqrt{\xi_{\alpha}}$ [lower panel (b)] holds in the linear phase. We can see that as expected [15], the growth rates of modes $\omega=7 \Omega_{\alpha}$ to $12 \Omega_{\alpha}$ scale as $\sqrt{\xi_{\alpha}}$. Mode $\omega=6 \Omega_{\alpha}$ does not follow this scaling and here marks the transition to the linearly stable regime.

The nonlinear simulations presented here resolve a longstanding question in the physics of fusion alpha-particle confinement and stability in MCF plasmas. They confirm the MCI as the likely emission mechanism and greatly strengthen the basis for diagnostic exploitation of ICE in future burning plasmas.

Our results suggest that a fast linear phase of the MCI deposits energy into the wave fields of all of the linearly unstable modes, and preferentially into the fastest growing modes $8 \leq l \leq 10$. It is these modes that dominate the power spectrum at the start of the nonlinear phase. Thus the system enters its nonlinear phase, which begins when the fastest growing mode saturates, imprinted with first, the energy acquired by each individual mode according to its particular growth rate, and second, the total energy transferred from the energetic ion population to the excited fields, which scales with $n_{\alpha} / n_{i}$. During the nonlinear phase this energy is redistributed across all the harmonics $1 \leq l \leq 12$.
The energy density of the fundamental and low cyclotron harmonic modes $(1 \leq l \leq 6)$, which are linearly stable, are driven up by nonlinear coupling. In the saturated nonlinear regime, wave energy density scales approximately linearly with $n_{\alpha}$ at each harmonic, regardless of its linear stability characteristics. Our simulations then suggest that, for the range of $n_{\alpha} / n_{i}$ considered, the relative amplitudes of the cyclotron harmonic peaks of the saturated power spectra are independent of $n_{\alpha} / n_{i}$ to within error bars. The linearly stable, but nonlinearly excited low cyclotron harmonic modes $(1 \leq l \leq 6)$ have peak intensity scaling that follows that established experimentally. The diagnostic exploitation of ICE in future deuterium-tritium plasmas rests on the empirical scaling between ICE signal intensity and fusion product concentration. The results presented here provide the first-principles physics basis for this observed scaling.

L. C. acknowledges the Mexican Council of Science and Technology (CONACyT) for support. This work was part funded by the RCUK Energy Program (under Grant No. EP/I501045) and the European Communities. We acknowledge the EPSRC for financial support. This work has been carried out within the framework of the EUROfusion Consortium and has received funding from the Euratom research and training program under Grant No. 633053. The views and opinions expressed herein do not necessarily reflect those of the European Commission. This was written by the authors acting in their own independent capacity and not on behalf of UT-Battelle, LLC, or its affiliates or successors.

*carbajalgoml@ornl.gov

[1] K. G. McClements, R. D’Inca, R. O. Dendy, L. Carbajal, S. C. Chapman, J. W. S. Cook, R. Harvey, B. Heidbrink, and S. Pinches, Nucl. Fusion 55, 043013 (2015).

[2] N. N. Gorelenkov, S. D. Pinches, and K. Toi, Nucl. Fusion 54, 125001 (2014).

[3] N. N. Gorelenkov, New J. Phys. 18, 105010 (2016).

[4] S. Cauffman, R. Majeski, K. G. McClements, and R. O. Dendy, Nucl. Fusion 35, 1597 (1995).

[5] G. A. Cottrell, V. P. Bhatnagar, O. Da Costa, R. O. Dendy, J. Jacquinot, K. G. McClements, D. C. McCune, M. F. F. Nave, P. Smeulders, and D. F. H. Start, Nucl. Fusion 33, 1365 (1993).

[6] R. O. Dendy, K. G. McClements, C. N. Lashmore-Davies, G. A. Cottrell, R. Majeski, and S. Cauffman, Nucl. Fusion 35, 1733 (1995).

[7] V. S. Belikov and Y. I. Kolesnichenko, Sov. Phys. Tech. Phys. 20, 1146 (1976).

[8] R. O. Dendy, C. N. Lashmore-Davies, K. G. McClements, and G. A. Cottrell, Phys. Plasmas 1, 1918 (1994).

[9] K. G. McClements, R. O. Dendy, C. N. Lashmore-Davies, G. A. Cottrell, S. Cauffman, and R. Majeski, Phys. Plasmas, 3, 543 (1996).

[10] T. Fülöp and M. Lisak, Nucl. Fusion 38, 761 (1998).

[11] T. Fülöp, M. Lisak, Ya. I. Kolesnichenko, and D. Anderson, Phys. Plasmas 7, 1479 (2000). 
[12] N. Gorelenkov and C. Z. Cheng, Nucl. Fusion 35, 1743 (1995).

[13] H. Smith and E. Verwichte, Plasma Phys. Controlled Fusion 51, 075001 (2009).

[14] J. W. S. Cook, R. O. Dendy, and S. C. Chapman, Plasma Phys. Controlled Fusion 55, 065003 (2013).

[15] L. Carbajal, R. O. Dendy, S. C. Chapman, and J. W. S. Cook, Phys. Plasmas 21, 012106 (2014).

[16] G. A. Cottrell and R. O. Dendy, Phys. Rev. Lett. 60, 33 (1988).

[17] K. G. McClements, C. Hunt, R. O. Dendy, and G. A. Cottrell, Phys. Rev. Lett. 82, 2099 (1999).

[18] W. W. Heidbrink, M. E. Austin, R. K. Fisher, M. GarcaMuoz, G. Matsunaga, G. R. McKee, R. A. Moyer, C. M. Muscatello, M. Okabayashi, D. C. Pace, K. Shinohara, W. M. Solomon, E. J. Strait, M. A. Van Zeeland, and Y. B. Zhu, Plasma Phys. Controlled Fusion 53, 085028 (2011).

[19] R. D'Inca, M. Garcia-Munoz et al., in Proceeding of the 38th EPS Conference Plasma Physics (Curran Associates, Inc., Strasbourg, France, 2012), p. 1.053.

[20] R. D'Inca, Ph.D thesis, Max Planck Institute for Plasma Physics, 2014.

[21] M. Ichimura, H. Higaki, S. Kakimoto, Y. Yamaguchi, K. Nemoto, M. Katano, M. Ishikawa, S. Moriyama, and T. Suzuki, Nucl. Fusion 48, 035012 (2008).

[22] S. Sato, M. Ichimura, Y. Yamaguchi, M. Katano, Y. Imai, T. Murakami, Y. Miyake, T. Yokoyama, S. Moriyama, T.
Kobayashi, A. Kojima, K. Shinohara, Y. Sakamoto, T. Watanabe, H. Hojo, and T. Imai, Plasma Fusion Res. 5, S2067 (2010).

[23] R. O. Dendy, K. G. McClements, C. N. Lashmore-Davies, R. Majeski, and S. Cauffman, Phys. Plasmas 1, 3407 (1994).

[24] K. Saito, R. Kumazawa et al., Plasma Sci. Technol. 15, 209 (2013).

[25] S. G. Thatipamula, G. S. Yun, J. Leem, H. K. Park, K. W. Kim, T. Akiyama, and S. G. Lee, Plasma Phys. Cont. Fusion (to be published).

[26] K. G. McClements and R. O. Dendy, J. Geophys. Res. 98, 11689 (1993).

[27] K. G. McClements, R. O. Dendy, and C. N. LashmoreDavies, J. Geophys. Res. 99, 23685 (1994).

[28] V. L. Rekaa, S. C. Chapman, and R. O. Dendy, Astrophys. J. 791, 26 (2014).

[29] J. W. S. Cook, S. C. Chapman, and R. O. Dendy, Phys. Rev. Lett. 105, 255003 (2010).

[30] PROMETHEUS++ code, http://www2.warwick.ac.uk/fac/sci/ physics/research/cfsa/people/carbajal_gomez/plasma_ modelling.

[31] P. W. Gingell, S. C. Chapman, R. O. Dendy, and C. S. Brady, Plasma Phys. Controlled Fusion 54, 065005 (2012).

[32] P. W. Gingell, S. C. Chapman, and R. O. Dendy, Plasma Phys. Controlled Fusion 56, 035012 (2014).

[33] R. D. Sydora, J. Comput. Appl. Math. 109, 243 (1999). 